\title{
REVISED FIBROMYALGIA IMPACT QUESTIONNAIRE (FIQR) USED AS A SCREENING TEST FOR MENTAL DISORDERS
}

\author{
Alessandra de Sousa Braz ${ }^{1,2, \star}$, Jordanne Duarte Passosㄹ, Luís Fábio Barbosa Botelho ${ }^{1,2,}$, Thainá Cavalcanti Mendes Pinto \\ 1.Universidade Federal da Paraíba, João Pessoa (PB), Brazil. 2.Hospital Universitário Lauro Wanderley, João Pessoa (PB), Brazil. \\ ${ }^{\star}$ Corresponding author: alessabraz@gmail.com
}

\section{BACKGROUND}

Fibromyalgia syndrome (FMS) is a disease characterized by the presence of chronic, multifocal, vigorous and strenuous musculoskeletal pain. It's closely related to psychiatric disorders, in which anxiety disorders and depression are the most prevalent of them. The purpose of this study is to evaluate whether the use of the Revised Fibromyalgia Impact Questionnaire (FIQR) - which contains a visual analogue scale (VAS) for anxiety and depression - as a screening test for anxiety and depression in patients with fibromyalgia is comparable to questionnaires general anxiety disorder - 7 (GAD-7) and Patient Health Questionnaire - 9 (PHQ-9), used as a screening test of such mental disorders in primary care.

\section{MATERIALS AND METHODS}

This is an observational, analytical-descriptive and cross-sectional quantitative study carried out between August 2019 and February 2020 at the fibromyalgia and chronic pain department of a tertiary-care hospital. The confidence interval was estimated at $99 \%$ and the level of significance was set at $p<0.01$. Spearman's correlation test was applied between variables referring to PHQ9 and VAS referring to the degree of depression and between GAD-7 and VAS referring to the degree of anxiety.

\section{RESULTS}

Participants were 84 patients, with an average age of 41 years, mostly female and $54.1 \%$ of the interviewees reported a pain syndrome for the last five years. There was a correlation of 0.66 with $p$ value $=8.29-12$ between the data collected through $\mathrm{PHQ} 9$ and the depression VAS score, proving a moderate to a strong correlation between the variables. Analyzing the variables referring to GAD-7 and the VAS score regarding the degree of anxiety, the correlation found was 0.61 with $p$ value $=9.36-10$, also demonstrating a moderate to a strong correlation between the variables.

\section{CONCLUSION}

There is an important correlation between the individual perception of the patients with FMS about his mental symptoms and a directed evaluation of his symptoms, conducted by specific instruments. In addition, it's possible to infer the good sensitivity of the VAS for the clinical evaluation of the patient with chronic pain. Thus, the application of FIQR in patients with chronic pain in primary and secondary services can be used as a screening test for anxiety and depression disorders with good reliability, allowing to separate patients who need more attentive mental care from those who have lower levels on the VAS, with a more physical condition than mental illness. 